\title{
Hystology findings' correlation between the ossicular chain in the transoperative and cholesteatomas
}

Cristina Dornelles ', Letícia Petersen Schmidt Rosito $^{2}$, Luíse Meurer ${ }^{3}$, Sady Selaimen da Costa ${ }^{4}$, Andréia Argenta ${ }^{5}$, Sabrina Lima Alves ${ }^{6}$
Keywords: ossicular chain, cholesteatoma, inflammation, chronic otitis media, perimatrix.

\section{Summary}

\begin{abstract}
C
hronic otitis media is hystopathologycaly defined as the presence of irreversible inflammatory tissue changes in the middle ear. Ossicular lesions represent the most prevalent change. Aim: to correlate the degree of ossicular chain changes seen during surgery with the inflammatory histological degree and the thickness of the cholesteatoma perimatrix. Study design: Cross-sectional study. Methods: Seventyone descriptions of surgeries done in patients submitted to tympanomastoydectomy were reviewed. Cholesteatoma were collected and fixed in 10\% formaldehyde. Two slides were made for each cholesteatoma, one stained with HE and another with picrossirius. Images were obtained from light microscopy and digitally processed and "blindly" analyzed using Image Pro-Plus Software. For statistical analysis we used Spearman's coefficient. Differences were considered statistically significant if $P \& \# 8804 ; 0.05$. Results: the ossicular chain was involved in 65 cases. The incus was the most frequently affected bone, followed by the stapes and the malleus. When the Spearman's coefficient was employed considering ossicular chain change degree with patient's age by the time of surgery, perimatrix thickness and histological degree of inflammation, correlations were not established. Conclusion: Our findings indicate that ossicular chain changes are practically universal when a cholesteatoma is present. We didn't find correlations related with bone erosion and cholesteatoma's histological findings.
\end{abstract}

${ }^{1}$ M.Sc. in Medical Sciences - Pediatrics, Doctorate student at Post-Graduation Course in Medical Sciences: Pediatrics - UFRGS, Biologist at the Brazilian Center for Otitis Media. Professor at IPA Post-Graduation Course in Speech Therapy. Invited Professor at the School of Medicine of UFRGS.

${ }^{2}$ Ear, Nose and Throat Physician, M.Sc. Student in Medical Sciences: Surgery.

${ }^{3}$ Doctor in Medical Sciences: Gastroenterology, Substitute Professor.

${ }^{4}$ Doctor in Surgery. Associate Professor.

${ }^{5}$ Scholar in Medicine.

${ }^{6}$ Scholar in Medicine.

Ear, Nose and Throat Service - Clínicas de Porto Alegre Hospital - Universidade Federal do Rio Grande do Sul. Mail address: Cristina Dornelles - Rua Cangussu 1343 Porto Alegre RS 90.830-010.

Fundo de Investimento em Pesquisa e Eventos do Hospital de Clínicas de Porto Alegre (FIPE-HCPA). (Research and Events Investment Fund of Clínicas de Porto Alegre Hospital)

Paper submitted to the ABORL-CCF SGP (Management Publications System) on August 29th, 2006 and accepted for publication on September 30th, 2006. cod. 3364. 


\section{INTRODUCTION}

The presence of cholesteatoma at the ear cleft of patients with chronic otitis media, undoubtedly causes more morbimortality due to the great bone erosion power of these epithelial accumulations. ${ }^{1,2}$

They usually reach the ossicular chain and in a lesser extent the skull bones. Even the most rigid bone in the human body, the optic capsule, is affected, showing its strong destroying action on the bone tissue. Partial or total destruction of ossicles is seen in approximately 80\% of patients with cholesteatoma, whereas in chronic otitis media without cholesteatoma, ossicular chain erosion can be seen in approximately $20 \%$ of the cases ${ }^{3}$. The mechanisms leading to this increase in bone degradation in the presence of cholesteatoma are still unclear. ${ }^{4}$ According to Swartz ${ }^{4}$, ossicular destruction is certainly the most common problem among cholesteatoma complications and the type of destruction depends on its origin and the way it spreads itself. According to his data, ossicular chain is intact in only $26 \%$ of attical cholesteatomas. The most affected region is the long process of the incus followed by the incus body and the malleus head. On the other hand, pars tensa cholesteatomas show an erosion power of $90 \%$.

Bone absorption is stimulated by several factors, including inflammation, local pressure and specific cytokeratins ${ }^{5}$. The enzymatic concept in which epithelial origin enzymes are considered to be responsible for bone destruction was defined by Abramson ${ }^{6}$, who showed the presence of collagenases and hydrolysis in cholesteatoma. This hypothesis was later confirmed by Thompsen ${ }^{7}$. Ferlito et al. ${ }^{8}$ suggested that the destructive property of cholesteatomas, bone erosion, is caused by the collagenases produced by the components of squamous and fibrous epithelial tissues. The hypothesis of bone absorption by biochemical action exclusively done by collagenolitic enzimes was later incorporated by other agents, such as tumor necrosis factor (TNF), interleukins (IL-1a) and prostaglandins (PGE2) ${ }^{9-11}$.

By the histologic analysis of cholesteatomas, Dornelles ${ }^{12}$ found an inverse correlation between the perimatrix size measured in micrometers and the age of patients at the day of surgery and that the degree of the perimatrix inflammation was strongly correlated with the perimatrix thickness. However, in this paper we did not evaluate the relationship between the degree of perimatrix inflammation and the ossicular involvement.

This piece of work wants to correlate the degree of the ossicular chain involvement seen at the trans-operative stage with the age of patients at the day of surgery, the histological degree of inflammation and the perimatrix thickness of acquired cholesteatomas.

\section{METHODOLOGY}

The Research and Post-Graduation Group of this institution approved this piece of work on its ethical and methodological aspects with the number 03-271 in 2003. Due to its methodology it is classified as a comparative and contemporary cross study.

We reviewed the descriptions of trans-operative findings of 71 patients with chronic otitis media with cholesteatoma (COMC), followed up at the Chronic Otitis Media outpatient clinic, submitted to tympanomastoidectomy between March 2003 and June 2006.

Surgical descriptions were written by the resident physician who was the first assistant at the surgeries, with data from patients, pathology, conduct and specially the inspection of the ossicular chain which is thoroughly detailed.

In order to quantify the degree of ossicular erosion the following scale was prepared adding up the score according to the items: 0 - if the ossicular chain is intact; 1 - for discontinuity of the chain; 1 - for each eroded ossicle; 2 - absence of ossicle; 3 - eroded footplate. This score was cumulative and in order to obtain the score of the patient every alteration found was added up, having as a result a scale with values between 0 and 10 .

Cholesteatomas were collected by the otologist surgeon, immediately fixed in formol at 10\%, processed by regular histological techniques and embedded in paraffin. For the morphological analysis of each sample two slides were prepared. The slides were stained in HematoxilineEosine (HE) and Picrossirius (Sirius Red) and analyzed in an optical microscope (Figure 1). Please note that in Figure 1 the contrast obtained in the second staining is much greater, since collagen fibers on them are stained in burgandy, which makes easier the differentiation of the perimatrix thickness to be measured. The reading of the material was "blind" and controlled by the researcher. The following constituents were observed: perimatrix thickness and inflammation.

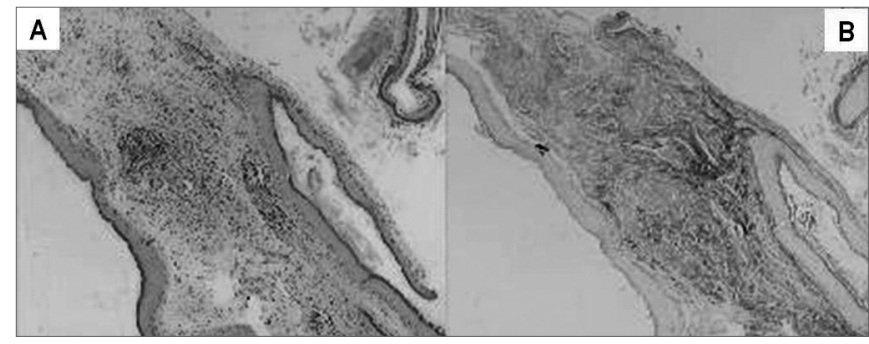

Figure 1. Same sample of stained cholesteatoma in HE (A) and Picrosirius (Sirius Red) (B). 
For the histological degree of inflammation, an ordinal variable was created with values from zero to three, where $0=$ absent, $1=$ discreet, $2=$ moderate and $3=$ accentuated, characterized by the intensity of perimatrix permeation by lymphocytes, neutrophils, plasmocites and macrophages ${ }^{13}$.

Perimatrix thickness was obtained through the analysis of computarized images using ImagePro Plus Media Cybernetics software (Figure 2). For each sample we took 20 perimatrix thickness measurements and they were summarized by the average. This was the parameter used to test the correlation of perimatrix thickness with the ossicular erosion degree.

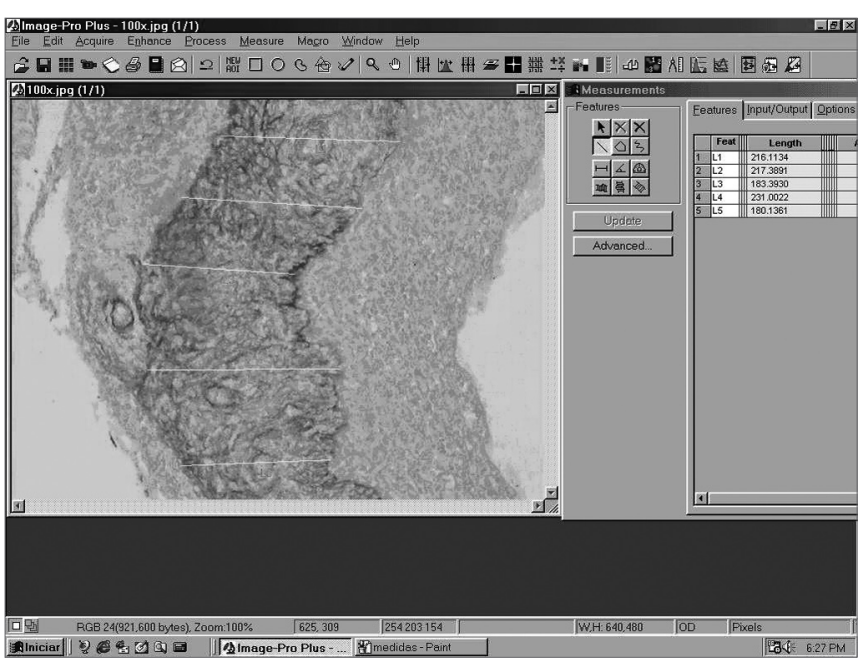

Figure 2. Screen image of ImagePro Plus Media Cybernetics software.

Patients were classified into two groups according to their age at the day of surgery, as follows: Pediatrics - patients no older than 18 years old and Adults - patients older than 19 years old.

For the statistical analysis, we used Spearman's coefficient in the analysis of the correlation of ossicular erosion degree with the histological degree of inflammation, with perimatrix thickness and age of patient at the day of surgery. For descriptive data we use frequency tables and the comparison between the groups was done through a Student's t- test, using SPSS 10.0 software for Windows. P values below 0.05 were considered as statistically significant.

\section{RESULTS}

\section{Epidemiological Data}

The sample had 71 patients with \pm standard deviation of $26 \pm 18$ years old. In this group we had 38 patients being 18 years old or younger with \pm standard deviation equal to $11 \pm 4$ years old. There were 33 patients older than 18 years old, with \pm standard deviation equal to $39 \pm 15$ years old.

\section{Histological findings}

Perimatrix appears as an inflammatory network surrounding the cholesteatoma with variable thickness both intra and inter-patients. The total group showed an average of 80 micrometers with interquartile interval of 37 to 232 , with the minimum value zero and the maximum value 1.926 .

When we analyzed inflammation degree at the perimatrix with the optical microscope, 60\% of the samples were classified as moderate and accentuated.

\section{Trans-operative Data and Correlations}

Ossicular chain was somehow compromised in 65 (92\%) of the 71 patients reviewed (Table 1).

Table 1. State of ossicular chain in pediatric and adult groups.

\begin{tabular}{lccc}
\hline \multicolumn{1}{c}{ State of the chain } & Pediatric & Adult & Total \\
\hline Complete & 2 & 4 & 6 \\
One eroded ossicle & 5 & 5 & 10 \\
Two eroded ossicles & 10 & 3 & 13 \\
$\begin{array}{l}\text { Three eroded ossicles } \\
\text { One eroded ossicle and }\end{array}$ & 7 & 5 & 12 \\
$\begin{array}{l}\text { one absent ossicle } \\
\begin{array}{l}\text { One eroded ossicle or two } \\
\text { absent ossicles }\end{array}\end{array}$ & 1 & 4 & 10 \\
$\begin{array}{l}\text { Two eroded ossicles or one } \\
\text { absent }\end{array}$ & 2 & 3 & 4 \\
$\begin{array}{l}\text { Two eroded ossicles and } \\
\text { stape footplate absent }\end{array}$ & 3 & 6 & 8 \\
Absence of ossicular chain & 2 & 2 & 4 \\
\hline Total & 38 & 33 & 71 \\
\hline
\end{tabular}

When we analyzed each ossicle separately, the incus was the most frequently affected, which was absent in $14.2 \%$ of procedures and with erosion of its long process in $47.6 \%$, followed by the stapes with erosion of its suprastructure in $32.3 \%$ and by malleus which was absent in $3.8 \%$ of the total number of ears.

The degree of compromise of the ossicular chain showed an average of $4.35 \pm 2.52$ in the sample, with 4.26 \pm 2.37 in pediatric patients and $4.45 \pm 2.73$ in the adult group. When comparing the degree of involvement of the ossicular chain between the age groups through the Student's t- test, we did not find any statistically significant difference $(\mathrm{P}=0.753)$. When we applied Spearman's coefficient between the degree of involvement of the ossicular chain and the age of the patient at the day of surgery, perimatrix thickness and the histological degree of inflammation, we did not find any correlation (Figure 3). 


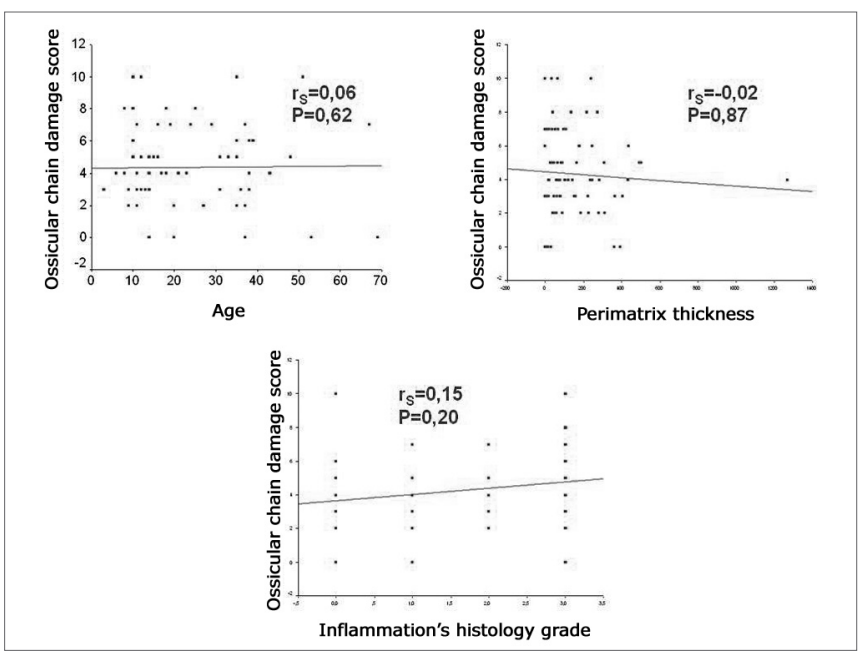

Figure 3. Scatter Plot between the degree of compromised ossicular chain and the age of patients at the day of surgery, perimatrix thickness and histological degree of inflammation.

\section{DISCUSSION}

Ossicular alterations take part in the concept of chronic otitis media, that is to say, irreversible inflammatory tissue damages. This type of involvement has a clear clinical repercussion since it affects the basis of sound air conduction to the inner ear, having as an unavoidable result a air conduction dysacusia of varied intensity. It is believed that the type of alteration caused by the chronic inflammatory process in the ossicular chain follows a pattern that tends to be continuous and more or less repetitive. This means that the ossicular structure is kept in a fragile balance between its own resistance and the destructive mechanisms of chronic inflammation.

Sadé and Fuchs ${ }^{14}$ compared ossicular erosion findings in adults with those found in children. Destruction percentage in stapes and malleus was similar in both groups; however, the incus shows a significantly higher destruction in adults. In this study we did not find any differences in the score of both ossicular damage and ossicles separately between pediatric and adult groups.

In a previous study of our groups ${ }^{15}$, we did a description study of the middle ear findings at the transoperative stage of 55 patients with chronic otitis media, followed up at the Chronic Otitis Media outpatient clinic. In this group of patients $49 \%$ had the diagnosis of Chronic Otitis Media with Cholesteatoma (COMC). In the sample as a whole, there was some involvement of ossicular chain in 66\%, in COMC this rate was 96\% and in Chronic Otitis Media without Cholesteatoma was reduced to $37 \%$. The presence of cholesteatoma was associated with the existence of two or more ossicles affected, as well as with the higher prevalence of absence or erosion of the ossicles. These findings indicate that most patients with COM, who underwent a surgical intervention has some impact in the ossicular chain and that the frequency and extension of this involvement were much more related to the presence of cholesteatoma.

In our study, the incus was the most affected ossicle by pathology, followed by the stapes and by the malleus respectively. The fact the incus is the most affected ossicle is maybe due to its incudal mass, to its prominent bone marrow and mainly, to the exposure and fragility of the long apophysis and its lenticular process. These factors acting synergically would make this ossicle more vulnerable to extrinsic aggressions and to osteomyelitis processes. These findings confirm those by Tos16, who reviewed ossicular pathology in 1.150 ears with chronic otitis media and indicated the incus, stapes and malleus sequence as the most frequently affected by the inflammatory process.

Today it is believed that ossicular defects are caused by active processes of bone resorption and not by ossicular necrosis. This theory presupposes the presence and participation of living cells at the demineralization, erosion and bone destruction mechanisms ${ }^{17}$. A simply necrotic bone can remain in situ for several years without suffering any resorption process. This possibility is well illustrated by the techniques of ossicular chain reconstruction with implants of homologous ossicles. In these situations, the ossicles are kept intact at the long run, allowing the propagation of sound stimulus through the middle ear.

Bone resorption mechanism in chronic otitis media is not completely understood. Ruedi ${ }^{18}$ and Tumarkin ${ }^{19}$ suggested that bone resorption would be due to the pressure placed by cholesteatomas on the ossicular surface. Thompsen et al. ${ }^{20}$ and Sadé \& Berco ${ }^{21}$ noticed that eroded ossicles were unvariably surrounded by an inflammatory reaction and they suggested that the inflammation was the cause of ossicular resorption. It has been proved that the granulation tissue adjacent to the ossicles is able to produce a variety of enzymes and mediators that accelerate ossicular resorption; they include lysosomic enzimes ${ }^{22}$, collagenases $^{23}$ and prostaglandins $s^{24}$. However, the dominating cell in bone resorption process still has controversies. Whereas some studies reveal the presence of osteoclasts in the areas of bone destruction ${ }^{24}$, others indicate mononuclear cells as being responsible for the situation ${ }^{25}$. According to some authors, the persistent inflammation on chronic otitis media with cholesteatoma would cause a constant healing process at the cholesteatoma perimatrix, with the consequent increase of cytokine levels. Among other factors, they could be responsible for cholesteatoma growth and for the bone destruction caused by $\mathrm{it}^{26}$.

In a previous study ${ }^{12}$, our group found several indications that the degree of histological inflammation would be strongly correlated with the perimatrix thickness of cholesteatomas. According to Mayot et $a{ }^{27}{ }^{27}$, in the case 
of chronic otitis media, the immune system defenses coming from the middle ear mucus would be recruited and would contribute to the pathogenia of the cholesteatoma. Therefore, perimatrix inflammation could contribute to the aberrant behavior of cholesteatoma ${ }^{28}$. Some authors believe that the granulation inflammatory tissue (perimatrix) needs bone erosion, which is a feature of the chronic otitis media with cholesteatoma. 4,29,30

We could draw an analogy between the perimatrix and a "battlefield", where there is a fight for the middle ear territory. On the one hand, on the attack we have the cholesteatoma; on the other hand, the adjacent tissues of the tympanic box mucus. With the expansion of cholesteatoma, the inflammatory reaction would increase and therefore, it would produce more elements of the inflammatory cascade.

This was the perspective we used to do this study, in order to verify the influence of perimatrix and its inflammatory state during the ossicular erosion process; different from what our hypothesis suggested, we did not find any correlation between the histological and trans-operative findings in our study cases.

Bone erosion resulting in chronic otitis media with cholesteatoma can be classified based on two hypothesis: biophysical and biochemical. Biophysical action basis would be the pressure done by the cholesteatoma on the ossicular chain and on the middle ear walls. On the other hand, the biochemical hypothesis is based on the destructive action of collagenases and inflammatory products.

With the methodology used in this study, we managed to do an indirect quantification of the biochemical action, although until now biophysical action does not have any methodology for measurement, therefore the fact that we did not find any correlation between the perimatrix thickness ( indirect measurement of collagenases) and the histological degree of inflammation (indirect measurement of inflammatory products) may not mean that it does not exist, but it could be influenced by the pressure factor of the cholesteatoma on the ossicular chain, that would work as a confusing factor on this analysis.

Another fact that may influence the results of this study is the long waiting time for surgery, since our patients came from the public health system. This means they had to face a waiting period of two to five years until surgery once the diagnosis was done. This long waiting time could be responsible for the advanced stage of ossicular erosion found on this sample.

Thus, cholesteatomas biology has to be better understood in order to be able to elucidate the real mechanisms that destroy the ossicular system. Studies on this issue are being done by our group through the immunohistochemical analysis of collagenases and angiogenesis.

\section{CONCLUSIONS}

Most patients with chronic otitis media with cholesteatomas who underwent surgery have some impact on the ossicular chain. It can be seen that ossicular destruction followed a trend of steps, beginning at the incus, then the stapes until reaching the malleus. All these findings corroborate those found in the literature.

We did not find any correlation between the ossicular erosion degree and the histological findings.

\section{ACKNOWLEDGMENTS}

We thank the Otolaryngology and Pathology Services at our institution for allowing us to use their infrastructure for the development of this project.

We also thank the Research and Post-Graduation Group at our institution for their technical support.

We thank the Research and Events Incentive Fund at our institution for the financial support.

\section{BIBLIOGRAPHICAL REFERENCES}

1. Sadé J, Halevy A. The etiology of bone destruction in chronic otitis media. J Laryngol Otol 1974;88:139-43.

2. Bretlau P, Jorgensens MB, Sorenses CH, Dabelsteen E. Bone resorption in human cholesteatomas. Ann Otol 1982;91:131-5.

3. Chole RA. The molecular biology of bone resorption due to chronic otitis media. Ann New York Acad Sci 1997;830:95-109.

4. Swartz J. D. Colesteatomas of the middle ear. Diagnosis Etiology and Complications. Radiol Clin North Am 1984;22:15-34.

5. Olszewska E, Wagner M, Bernal-Sprekelsen M. Ebmeyer J, Dazert S, Hildmann H, Sudhoff H. Etiopathogenesis of cholesteatoma. Eur Arch Otorhinolaryngol 2004;261:6-24.

6. Abramson M, Huang CC. Localization of collagenase in human middle ear cholesteatoma. Laryngoscope 1976;86:771-91.

7. Thompsen J. Bone resorption in chronic otitis media. In: McCabe BF, Sade J e Abramson M eds. Cholesteatoma: first international conference. Aescupulus Publishing Co.: Birmingham, ALA; 1977. p. 136.

8. Ferlito O, Devaney K.O, Rinaldo A, Milroy C, Wenig B, Iurato S, Mccabe BF. Clinicopathological consultation ear cholesteatoma versus cholesterol granuloma. Ann Otol Rhinol Laryngol 1997;106:79-85.

9. Minotti AM, Kountakis SE, Leighton WR, Cabral FR. Effects of extracellular calcium on cholesteatoma migration and adhesion in vitro. Otolaryngol Head Neck Surg 1996;115:458-63

10. Kurihara A, Toshima M, Yuasa R, Takasaka T. Bone destruction mechanisms in chronic otitis media with cholesteatoma: specific production by cholesteatoma tissue in culture of bone-resorbing activity attributable to interleukin-1 alpha. Ann Otol Rhinol Laryngol 1991;100:989-98.

11. Hansen T, Unger R.E, Gaumann A. Hundorf I, Maurer J, Kirkpatrick J. Kriegsmann J. Expression of matrix-degrading cysteine proteinase cathepsin $\mathrm{K}$ in cholesteatoma. The United States and Canadian Academy of Pathology 2001;14:1226-31.

12. Dornelles D. Colesteatomas Adquiridos: Análise Comparativa da Perimatriz entre Pacientes Pediátricos e Adultos. Dissertação de Mestrado. Faculdade de Medicina UFRGS; 2004.

13. Baak JPA. Manual of quantitative pathology in cancer diagnosis and prognosis. Springer-Verlag; 1991.

14. Sadé J, Fuchs C. Cholesteatoma: ossicular destruction in adults and children. J Laryngol Otol 1994;108:541-44. 
15. Dornelles C, Weber R, Schimdt VB, Schimdt L, Dall`Igna D, Carvalhal LHSK, Kruse L, Costa SS. Descrição da cadeia ossicular no transoperatório de pacientes com otite média crônica. Pesquisa: Logos e Práxis. Unidade de Pesquisa do Instituto de Cardiologia; 2002. p. 221.

16. Tos M. Pathology of the ossicular chain in various chronic middle ear diseases. J Laryngol Otol 1979;93:769-80.

17. Krane SM, Dayer JM, Goldring SR. Ossicular pathology In: McCabe BF, Sadé J, Abramsow M. Cholesteatoma First International Conference. Birmingham: Ausculapios; 1977. 102-10.

18. Ruedi L. Cholesteatosis of attic. Laryngol Otol 1958;73:593-609.

19. Tumarkin A. Attic cholesteatoma. J Laryngol Otol 1958;72:610-19.

20. Thompsen J, Jorgensen B, Bretlau P, Kristensen HK. Bone resorption in chronic otitis media. A histological and ultrastructural study. I. Ossicular necrosis. J Laryngol Otol 1974;88:975-82.

21. Sadé J, Berco E. Bone destruction in chronic otitis media. A histopathological study. J. Laryngol Otol 1974;88:413-22.

22. Thompsen J, Bretlau P, Krintensen HK. Bone resorption in chronic otitis media: a light-microscopical and histochemical investigation of acid phosphatase activity. Acta Otolaryngol 1975;79:400-8.

23. Abransom M, Huang C. Localization of colagenase in human middle ear cholesteatoma. Laryngoscope 1977;87:771-91.
24. Bernstein JM, Hausmann E, Wright J. Middle ear disease release of soluble factor(s) stimulating bone resorptiom. In: McCabe BF, Sadé J, Abramson M. Cholesteatoma: First International Conference. Birmingham: Aesculapius Publishing Co.; 1977. p.152-61.

25. Grippaudo M. Histopathological study of the ossicles in chronic otitis media. J Laryngol Otol 1958;72:177-89.

26. Milewski C. Role of perimatrix fibroblast in development of acquired middle ear cholesteatoma. HNO 1998;46:494-501.

27. Mayot D, Bénè MC, Faure GC, Wayoff M, Perrin C. Immunohistologic analysis of the cholesteatoma matrix in children. Int $\mathrm{J}$ Pediatric Otorhinolaryngol 1991;22:115-24

28. Palva A, Karma P, Kärjä J. Cholesteatoma in children. Arch Otolaryngol 1997;103:74-7.

29. Mafee M. MRI and CT in the evaluation of acquired and congenital cholesteatomas of temporal bone. J Otolaryngol 1993;22:239-48. In: Tessa JRG, Vivente AO, Abreu CEC, Bendassat SF, Antunes ML, Barros FA. Colesteatoma causando paralisia facial. Rev Bras Otorrinolaringol 2003;69:657-62.

30. Jung J, Chole R. Bone resorption in chronic otitis media: the role of the osteoclast. ORL 2002;64:95-107. 